\title{
Estimating strain and rotation from wrapped SAR interferograms
}

\author{
Alessandro Parizzi, Wael Abdel Jaber
}

\begin{abstract}
This letter aims to discuss a general framework that allows the direct interpretation of the wrapped DInSAR phase in terms of surface strain $S$ and rotation $R$ components. The methodology is demonstrated showing the estimation of strain and rotation components of a glacier flow using three TerraSAR-X interferometric geometries (ascending right-looking, descending right-looking and descending left-looking. Finally since the left looking geometry can be difficult to obtain on a regular basis, the surface parallel flow assumption is extended to the phase gradients inversion in order to reduce the amount of necessary geometries from three to two.
\end{abstract}

Index Terms-Differential SAR Interferometry, Frequency Estimation, gradient tensor, glaciers flow, strain, rotation.

\section{INTRODUCTION AND MOTIVATION}

Interferometric measurements can be directly related to the spatial gradients of the motion [1] [2] [3] [4]. This work exploits this property to resolve between strains and rotation components without the need to unwrap the interferometric phase. The paper deals first with the theoretical framework: the direct problem is described showing the link between the gradient tensor and the interferometric phase gradients. The inversion is then discussed in the general case of $N$ available geometries. In Section III it is shown how the interferometric measurements, necessary for the problem inversion, can be obtained by estimating the local fringe frequency, avoiding therefore phase unwrapping [5]. Finally the method is tested on Darwin Glacier (Antarctica). Strain and rotation components are inverted in two scenarios: first using three interferometric LoSs (Line of Sight) then using two LoSs and assuming surface parallel flow. The study of a glacier flow has been used as test case for the methodology since the measurement of strains is a relevant topic for glaciology [6]. However the use of the interferometric phase in glaciological applications is limited by the large amount of motion which makes phase unwrapping very challenging. Therefore such measurements are presently computed mainly using correlation techniques [7]. This work proposes a methodology that, by relying on more than one acquisition geometry, exploits directly interferometric phase avoiding the phase unwrapping. Furthermore improving also significantly the estimation accuracy. This is due to the inherent frequency-bandwidth gain deriving from the coherent processing. Such gain can be computed from cross-correlation and interferometric phase accuracy expressions $\left(\sigma_{c o r r}^{2}, \sigma_{\phi}^{2}\right)$. Being $f_{0}$ the central frequency of the sensor and $B$ the signal bandwidth such ratio is $\frac{\sigma_{\text {corr }}^{2}}{\sigma_{\phi}^{2}}=\frac{12 f_{0}^{2}}{B^{2}}$ [8] assuming the measurements resolution the same. The aforementioned accuracy ratio is considerable, $50 d B$ for Sentinel IW mode. For PALSAR-2 Stripmap mode between 34 and $43 d B$ and about $47 d B$ in the specific case of the TerraSAR-X Stripmap used for this study.

\section{GRADIENT TENSOR FROM INSAR MEASUREMENTS}

Considering a SAR interferogram, where all the topographic components have been compensated and neglecting the atmospheric delay, the expression of the absolute interferometric phase will be:

$$
\phi=\frac{4 \pi}{\lambda} \boldsymbol{\delta} \cdot \boldsymbol{s}
$$

where $\lambda$ is the wavelength, $(e, n, v)$ the reference system oriented in accordance with the local east, the local north and the geodetic vertical. The change in radar range can be written as the projection of the displacement vector $\boldsymbol{\delta}=\left[\delta_{e}, \delta_{n}, \delta_{v}\right]$ on the sensing direction of the radar $s$. In order to be able to separate the strain and rotation components the gradient tensor $\Psi$ has to be estimated [9]. The gradient tensor $\Psi^{1}$ represents the derivatives of $\delta$.

$$
\boldsymbol{\Psi}=\left[\begin{array}{cc|c}
u_{e e} & u_{e n} & u_{e v} \\
u_{n e} & u_{n n} & u_{n v} \\
u_{v e} & u_{v n} & u_{v v}
\end{array}\right]
$$

Since a single SAR interferometric pair is not able to provide resolution/sampling in the third dimension [10], it is not possible to perform derivative measurements along the $v$ axis. Therefore the third column of the gradient tensor can not be observed. Hence the displacement gradients observed in the sensing direction of the radar can be written as:

$$
\vec{\nabla}(\boldsymbol{\delta} \cdot \boldsymbol{s})=\boldsymbol{\Psi}^{T} \cdot \mathbf{s}=\frac{\lambda}{4 \pi} \vec{\nabla} \phi
$$

Equation (3) is a vector providing the derivatives of $\delta \cdot s$ w.r.t. the north and the east directions. Hence to retrieve the six components of $\Psi$ at least three diverse interferometric measurements are needed.

\section{A. Problem Inversion}

In Equation (3) the direct problem that links the phase gradients measurements with the gradient tensor $\boldsymbol{\Psi}$ has been defined. Let us now suppose that in a general case we have $N$ gradient measurements in $N$ different geometries. $C_{d, k}$ is the covariance matrix relative to the $k^{t h}$ acquisition geometry, $\Psi$ can be inverted in the Least Square sense by minimizing the figure of merit $M$ w.r.t. each $u_{i j}$ :

\footnotetext{
${ }^{1}$ For the sake of simplicity, the notation $u_{i j}=\partial \delta_{i} / \partial j$ will be used to identify the derivatives from now on.
} 


$$
\begin{gathered}
M=\sum_{k=0}^{N-1}\left(\frac{\lambda_{k}}{4 \pi} \vec{\nabla} \phi_{k}-\boldsymbol{\Psi}\left(u_{i j}\right)^{T} \cdot \mathbf{s}_{k}\right)^{T} C_{d, k}^{-1} \\
\left(\frac{\lambda_{k}}{4 \pi} \vec{\nabla} \phi_{k}-\boldsymbol{\Psi}\left(u_{i j}\right)^{T} \cdot \mathbf{s}_{k}\right)
\end{gathered}
$$

The inversion is completely analogous to the inversion of the 3D displacement vector [11] [12] [13]. Therefore all the considerations regarding the performance with diverse acquisition geometries are valid also for the inversion of Equation (3).

\section{B. Separation between strain and rotation components}

$\Psi$ represents the first order Taylor approximation of the displacement field and it contains the information about all the relative movements of the portion of surface which it represents. The gradient tensor can be then decomposed in its symmetrical and anti-symmetrical parts. Those are respectively the strain $\boldsymbol{S}$ and rotation $\boldsymbol{R}$ components [9].

$$
\begin{aligned}
& \boldsymbol{S}=\frac{1}{2}\left(\boldsymbol{\Psi}+\boldsymbol{\Psi}^{T}\right)=\left[\begin{array}{cc|c}
u_{e e} & \frac{u_{e n}+u_{n e}}{2} & \frac{u_{v e}}{2} \\
\frac{u_{n e}+u_{e n}}{2} & u_{n n} & \frac{u_{v n}}{2} \\
\hline \frac{u_{v e}}{2} & \frac{u_{v n}}{2} & 0
\end{array}\right] \\
& \boldsymbol{R}=\frac{1}{2}\left(\Psi-\boldsymbol{\Psi}^{T}\right)=\left[\begin{array}{cc|c}
0 & \frac{u_{e n}-u_{n e}}{2} & -\frac{u_{v e}}{2} \\
\frac{u_{n e}-u_{e n}}{2} & 0 & -\frac{u_{v n}}{2} \\
\hline \frac{u_{v e}}{2} & \frac{u_{v n}}{2} & 0
\end{array}\right]
\end{aligned}
$$

Since the derivatives w.r.t. the $v$ direction $\left(u_{e v}, u_{n v}, u_{v v}\right)$ are missing (see Equation (2)), only the $2 \times 2$ upper part of $\boldsymbol{S}$ and $\boldsymbol{R}$ tensors can be properly characterized. Consequently the third line and the third column of $\boldsymbol{S}$ and $\boldsymbol{R}$ end up being basically the same. This in practice means that even if the sensor is sensitive to the gradients of the vertical motion, it is not possible to resolve between a vertical tilt and a vertical extension/compression. It is worth mentioning that according to the theory [9] some assumptions could be made allowing the observation of more components of $\boldsymbol{\Psi}$ or reducing the set of equations needed and also improving the estimation accuracy. For instance assuming that the ice is incompressible allows us to impose the first invariant of the strain tensor $I_{S} \equiv$ $\operatorname{Tr}(\boldsymbol{S}) \equiv 0$ deriving consequently also $u_{v v}$ [9] [7]. Setting $S \equiv 0$ the problem reduces to a rotation only estimation [14]. However the paper is mainly methodological therefore the aim is to present the problem in a general fashion. Case specific considerations are left for application studies.

\section{Phase Gradients estimation avoiding Phase UNWRAPPING}

In the previous Section the general framework of the estimation of the strain and rotation components was outlined. It is now necessary to discuss how the gradient measurements $\vec{\nabla} \phi_{k}$ can be estimated from the complex interferograms. The interferogram is a complex number $z(r, a)=$ $A(r, a) \exp (j \phi(r, a))$ where $A$ is the product of master and slave reflectivity, $(r, a)$ are the range and azimuth coordinates in radar geometry and $\phi$ is the interferometric phase as in Equation (1). Since in the case of glacier flow the interferometric phase varies by several wavelengths within the scale of the deformation pattern, it is possible to perform a local linear approximation of the deformation phase. Hence at a given point $\left(r_{0}, a_{0}\right)$ the interferogram $z$ can be substituted by its first order Taylor approximation:

$$
z \approx A \exp \left(j \frac{4 \pi}{\lambda}\left(\frac{\partial \delta_{s}}{\partial r}\left(r-r_{0}\right)+\frac{\partial \delta_{s}}{\partial a}\left(a-a_{0}\right)\right)+\phi_{0}\right)
$$

where $\delta_{s}=\delta \cdot s$ is the projection of the displacement vector along the line of sight. Equation (6) shows how the problem is basically the estimation of the main local fringe frequency of the interferogram. The problem can be solved in different ways with varying performance. Typically the periodogram estimator is used [15], however this estimator assumes flat spectrum which is not the case for the interferograms. Moreover in the case of very strong fringe patterns, such an estimator does not take into account the spectral shift effect [16], hence reducing the estimation performance. In such a case the ML approach proposed in [17] would be more suitable. In order to preserve the original interferometric information, the estimation of the main fringe frequency has to be performed directly from the single look interferogram in radar geometry. This does not match with the direct problem stated in Equation (3). A local transformation based on orbital information must hence be established between the geographic reference system used in the previous section and the radar coordinates. Let $\boldsymbol{T}$ be the rotation matrix representing such a transformation. $\widetilde{\vec{\nabla}} \phi$ and $\widetilde{C_{d}}$, the phase gradients and their covariance matrix calculated in radar geometry, can be recomputed in the local reference system as:

$$
\begin{aligned}
\vec{\nabla} \phi & =\boldsymbol{T}^{T} \widetilde{\overrightarrow{\vec{\nabla}} \phi} \\
C_{d} & =\boldsymbol{T}^{T} \widetilde{C_{d}} \boldsymbol{T}
\end{aligned}
$$

Figure 1 shows an example of gradient estimation from a single look interferogram for Darwin Glacier in Antarctica. The two figures basically represent two of the six equations in (4) measured w.r.t. the radar coordinates $(\widetilde{\vec{\nabla}} \phi$ in Equation 7).

\section{Strain and Rotation of ICE Flow: Example of DARWIN GLACIER}

As an illustration of the application of the proposed method, the estimation of the surface strain and rotation of the Darwin Glacier has been investigated. Darwin Glacier $\left(79^{\circ} 53^{\prime} S\right.$ $159^{\circ} 00^{\prime} E$ ) is an outlet glacier of the Transantarctic Mountains draining ice from the East Antarctic Ice Sheet into the Ross Ice Shelf. It flows in the east direction with a velocity of several tens of meters per year [11] between the Britannia Range and the Darwin Mountains to the south and the Cook Mountains to the north (see Figure 2). The analysis focuses on the overlapping area of the three acquisition geometries covering the mid-section of the glacier including the junction with its tributaries Hatherton and Touchdown Glacier. Both 


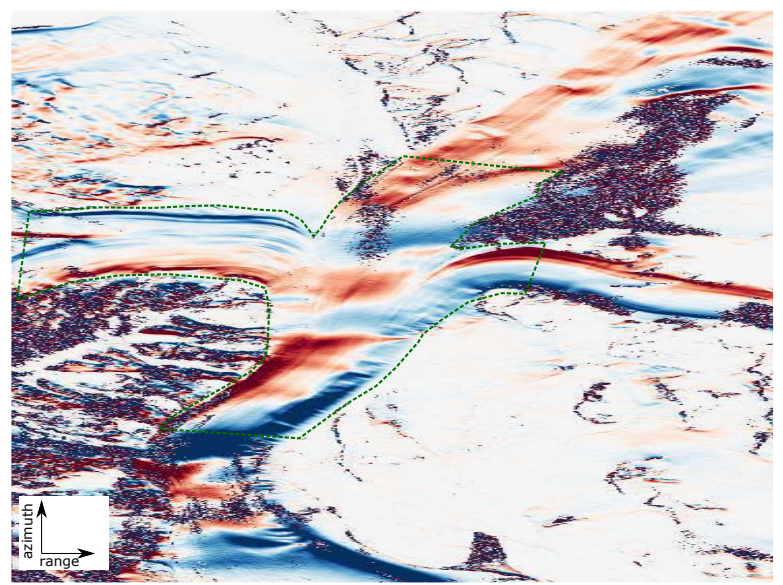

(a)

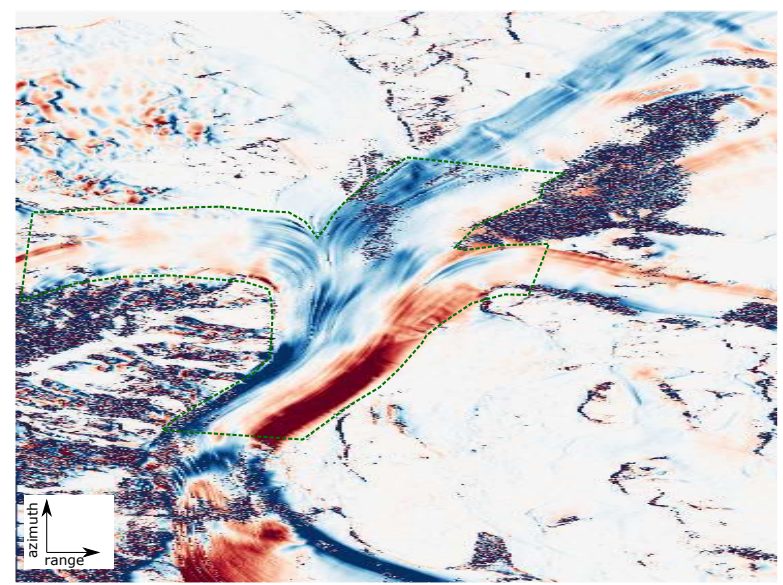

(b)

$[\mathrm{cm} / \mathrm{km}]$

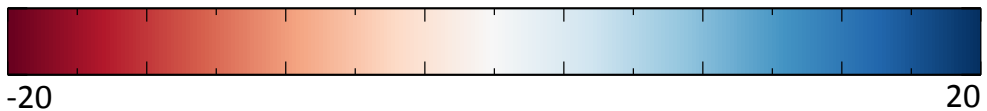

Fig. 1: Estimated gradients in radar geometry for the descending right-looking data-set. The AOI is highlighted by the dashed line. (a) Azimuth gradients (b) Range gradients.

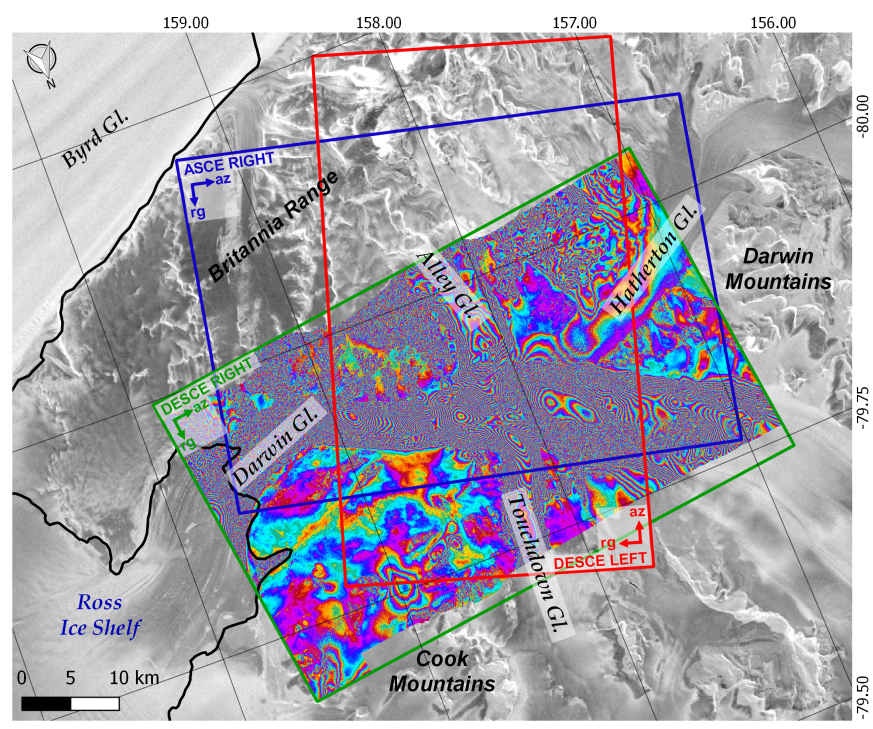

Fig. 2: Overview of the study area. The image shows the descending right-looking interferogram (20-APR-17 01-MAY17) the footprints of the three geometries and the grounding line. [18]

tributaries feature a slower ice flow as can be seen from the descending right-looking interferogram in Figure 2. The strain $\boldsymbol{S}$ and rotation $\boldsymbol{R}$ components have been firstly estimated using all three available geometries. The same inversion has been subsequently performed with two geometries only assuming the ice is flowing parallel to the glacier surface. A comparison of the two approaches follows at the end of the Section.

\section{A. Inversion using three acquisition geometries}

The framework proposed in the previous sections can support glaciological studies aimed at measuring and resolving the strain and rotation components of an ice flow. As previously mentioned, three different acquisition geometries are needed in order to be able to retrieve the six elements of $\Psi$. Currently SAR missions observe a given area on the Earth with ascending and descending geometries only. In cases where SAR footprints from different tracks strongly overlap, a third geometry is available for the inversion, nevertheless such a configuration has proved to be ill conditioned [13]. Hence left looking or squinted acquisitions have to be used in order to achieve a sufficient inversion accuracy [11] [13]. For this experiment three TerraSAR-X stripmap interferometric pairs have been acquired in ascending right-looking and descending right/left-looking (coverage is shown in Figure 2). Acquisitions dates and geometries are summarized in Table I. The temporal distribution of the acquisitions is optimal since they have the minimal temporal baseline between the interferometric acquisitions, 11 days between the pairs and 1 day between the different geometries. Therefore the observed motion can be assumed to be the same. Given the high latitude, the acquisitions result in high geometrical diversity providing good conditioning for the inverse problem. The TanDEM-X DEM [19] with posting of 0.8 arcsec in latitude and 0.4 arcsec in longitude was also available and has been used for co-registration and topographic phase removal. After the interferometric processing the LoS gradients were computed using estimation windows of about $180 m \times 180 m$ sampled on a $90 m \times 90 m$ raster (half a window overlap). As previously discussed three LoSs are sufficient for a constraintfree inversion of the problem in Equation (3). The components of the gradient tensor $\boldsymbol{\Psi}$ have then been inverted w.r.t. the 
TABLE I

DARWIN GLACIER TERRASAR-X ACQUISITIONS

\begin{tabular}{cccc}
\hline \hline & & & \\
Orbit & Look Dir. & $\theta_{\text {inc }}$ & Date \\
\hline & & & \\
ASCE & right & $28^{\circ}$ & 19-APR-2017 \\
DESCE & right & $31^{\circ}$ & 20-APR-2017 \\
DESCE & left & $46^{\circ}$ & 21-APR-2017 \\
ASCE & right & $28^{\circ}$ & 30-APR-2017 \\
DESCE & right & $31^{\circ}$ & 01-MAY-2017 \\
DESCE & left & $46^{\circ}$ & 02-MAY-2017 \\
& & & \\
\hline \hline
\end{tabular}

geographic directions $N / S$ and $E / W$ and the tensors $\boldsymbol{S}$ and $\boldsymbol{R}$ have been computed as shown in Equation (5). In order to have a more intuitive measurement unit the strain is displayed in $[\mathrm{cm} / \mathrm{km}]$ and the rotation in [arcsec] $\left(1 \operatorname{arcsec} \approx 0.0002^{\circ}\right)$. Results are shown in Figure 3.

\section{B. Inversion using two acquisition geometries assuming sur- face parallel flow}

Unfortunately no SAR mission currently performs observation campaigns in left or squinted geometry on a regular basis. It is hence worth discussing some assumptions that allow the removal of two of the six equations in (4). The idea is to exploit the information provided by an external DEM by assuming that ice flows parallel to the surface [20] [21]. The topography profile is approximated with the plane tangent to the DEM and the displacement is imposed to lie on that plane. This can be done by fitting a plane to the DEM samples surrounding the area of interest. The plane can be fully characterized by its normal $\boldsymbol{n}_{D}$. It is then possible to set $\boldsymbol{\delta} \cdot \boldsymbol{n}_{D}=0$ and differentiate w.r.t. $e, n$ :

$$
\vec{\nabla}\left(\boldsymbol{\delta} \cdot \boldsymbol{n}_{D}\right)=\boldsymbol{\Psi}^{T} \cdot \boldsymbol{n}_{D}=\mathbf{0}
$$

Since the topography slope is assumed constant in the estimation window it is possible to extract two more equations to retrieve the six parameters. For the sake of completeness it has to be noted that now the equation system depends also on the quality of external data. This means that a covariance matrix $C_{d, D E M}$ has to be derived from the DEM error map to characterize the error of the two equations coming from (8). The ascending/descending right-looking TerraSAR-X data described in Table I have been used for this experiment. The TanDEM-X DEM used for the interferometric processing has also been used to determine the local perpendicular to the topography. A comparison of the 3 and 2 LoSs inversions is given in Figure 3 (a) (c) and (d). The results look spatially smooth except for an area that is decorrelated ,probably due to the lack of backscatter intensity. The results obtained with the two approaches agree very well as can be seen from the difference plot (Figure 3-d).

\section{CONCLUSIONS}

The presented methodology extracts LoS projected gradients information from wrapped SAR interferograms and invert the observable part of the gradient tensor. From this description of the motion it is then possible to resolve between the gradient related to the surface deformation (strain) and the gradient representing a change in the orientation only (rotation). Since the measurements have no resolution along the vertical direction no information about the gradients of the vertical motion is directly available. Hence even if the vertical motion is very well captured by SAR interferometry it will never be possible (without models/assumptions) to determine whether such movement is triggered by tilting or vertical deformation (compressions/extensions) of the observed surface. The framework has been tested using three interferometric geometries estimating the deformation of glacier flow. Moreover the surface parallel flow assumption used in previous publications [20] [21] has been extended to the case of gradients tensor estimation. The results show good agreement between the two inversions however more glaciology-related assumptions should be discussed and tested in a more applicative context. Future work should also consider a validation campaign using in-situ measurements.

\section{ACKNOWLEDGMENT}

The authors would like to thank Dr. Dana Floricioiu for providing the TerraSAR-X data, Dr. Ramon Brcic for revising the manuscript, Prof. Angelika Humbert and Dr. Francesco De Zan for the fruitful discussions.

\section{REFERENCES}

[1] M. Facchini and P. Zanetta, "Derivatives of displacement obtained by direct manipulation of phase-shifted interferograms," Appl. Opt., vol. 34, no. 31, pp. 7202-7206, Nov 1995. [Online]. Available: http://ao.osa.org/abstract.cfm?URI=ao-34-31-7202

[2] D. Sandwell and E. Price, "Phase gradient approach to stacking interferograms," Journal of Geophysical Research, vol. 103, pp. 30,183-30,204, December 1998.

[3] A. Sharov, "Gradient Approach to InSAR modelling of glacial dynamics and morphology," Proc. 22nd EARSeL Symp., vol. Millpress, Rotterdam, pp. 373-381, 2003.

[4] R. R. Forster, K. C. Jezek, L. Koenig, and E. Deeb, "Measurement of glacier geophysical properties from InSAR wrapped phase," IEEE Transactions on Geoscience and Remote Sensing, vol. 41, no. 11, pp. 2595-2604, Nov 2003.

[5] A. Parizzi and M. Eineder, "InSAR Fringe Frequency and Deformation Gradient: application for Geophysical Modelling." Presented at EGU General Assembly, Wien, 2015.

[6] J. Nye, "The mechanics of glacier flow," Journal of Glaciology, pp. 82-93, 1952.

[7] R. Michel and E. Rignot, "Flow of Glaciar Moreno, Argentina, from repeat-pass Shuttle Imaging Radar images: comparison of the phase correlation method with radar interferometry," Journal of Glaciology, vol. 45 , no. 149 , p. 93100

[8] R. Bamler and M. Eineder, "Accuracy of differential shift estimation by correlation and split-bandwidth interferometry for wideband and delta-k SAR systems," IEEE Geoscience and Remote Sensing Letters, vol. 2, no. 2, pp. 151-155, April 2005.

[9] C. J. V. der Veen, Fundamentals of Glacier Dynamics, T. . F. Group, Ed. CRC Press, 2013.

[10] F. Banda, J. Dall, and S. Tebaldini, "Single and Multipolarimetric PBand SAR Tomography of Subsurface Ice Structure," IEEE Transactions on Geoscience and Remote Sensing, vol. 54, no. 5, pp. 2832-2845, May 2016.

[11] T. J. Wright, B. E. Parsons, and Z. Lu, "Toward mapping surface deformation in three dimensions using InSAR," Geophysical Research Letters, vol. 31, 2004.

[12] L. Gray, "Using multiple RADARSAT InSAR pairs to estimate a full three-dimensional solution for glacial ice movement," Geophysical Research Letters, vol. 38, no. 5, pp. n/a-n/a, 2011, 105502. [Online]. Available: http://dx.doi.org/10.1029/2010GL046484 


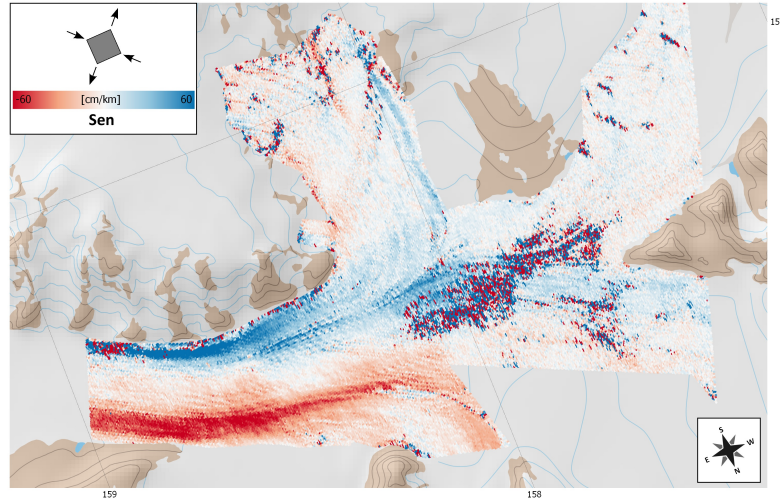

(a)

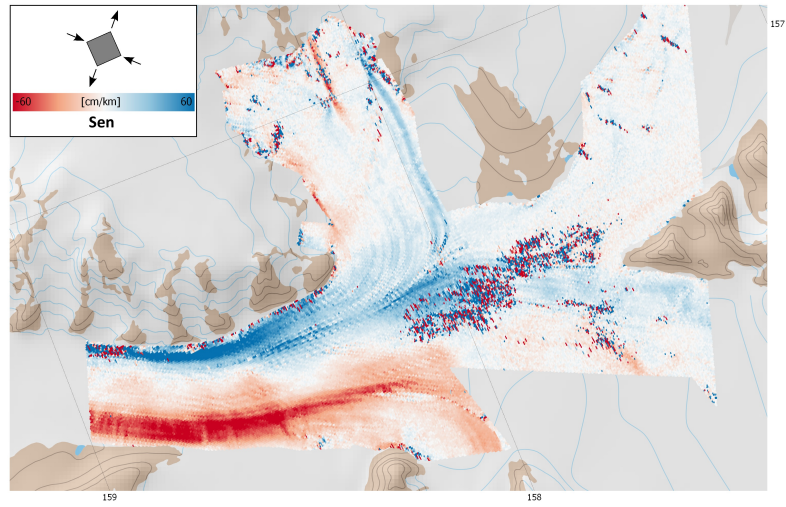

(c)

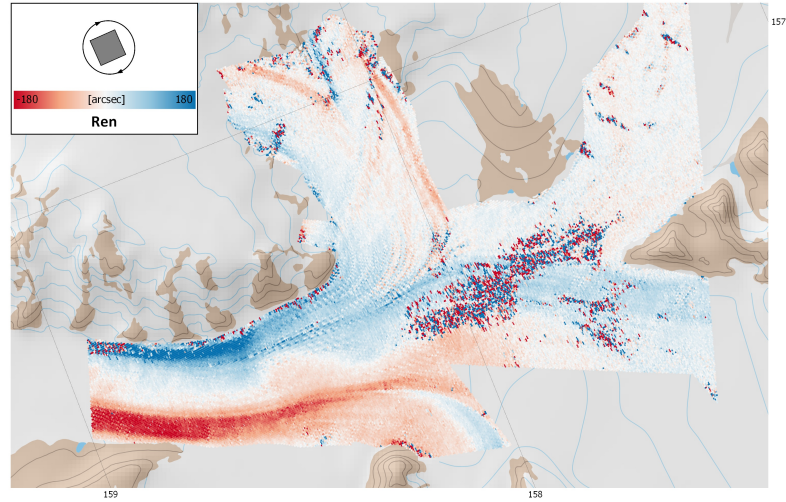

(b)

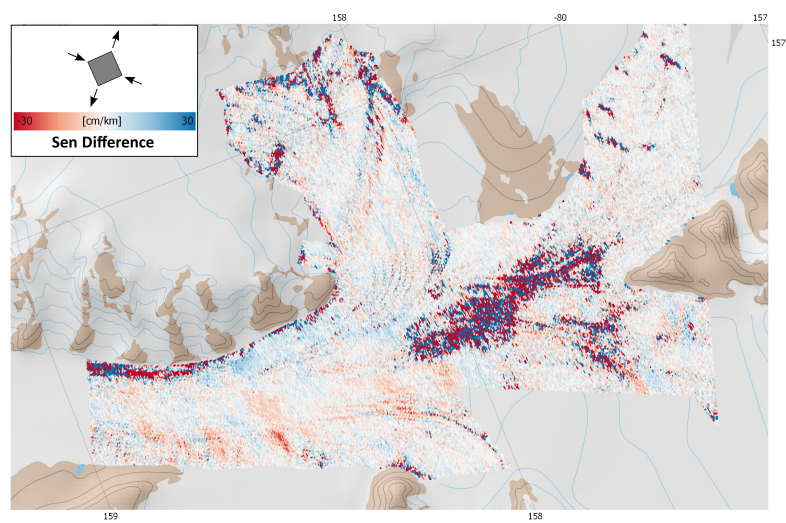

(d)

Fig. 3: Separation of the estimated gradient tensor in strain and rotation components. (a) shear component $\left(\boldsymbol{S}_{1,2}\right)$ estimated using three LoSs, (b) rotation component $\left(\boldsymbol{R}_{1,2}\right)$ estimated using three LoSs, (c) shear component $\left(\boldsymbol{S}_{1,2}\right)$ estimated using two LoSs and assuming surface parallel flow, (d) difference map between (a) and (c) $\left(\boldsymbol{S}_{1,2}^{3 \operatorname{LoS}}-S_{1,2}^{2 \operatorname{LoS}}\right)$

[13] H. Ansari, F. D. Zan, A. Parizzi, M. Eineder, K. Goel, and N. Adam, "Measuring 3-D Surface Motion With Future SAR Systems Based on Reflector Antennae," IEEE Geoscience and Remote Sensing Letters, vol. 13, no. 2, pp. 272-276, Feb 2016.

[14] P. López-Dekker, P. Prats, F. D. Zan, D. Schulze, G. Krieger, and A. Moreira, "TanDEM-X First DEM Acquisition: A Crossing Orbit Experiment," IEEE Geoscience and Remote Sensing Letters, vol. 8, pp. 943-947, 2011.

[15] S. Kay and R. Nekovei, "An efficient two-dimensional frequency estimator," Acoustics, Speech and Signal Processing, IEEE Transactions on, vol. 38, no. 10, pp. 1807-1809, Oct 1990.

[16] F. Gatelli, A. Monti Guamieri, F. Parizzi, P. Pasquali, C. Prati, and F. Rocca, "The wavenumber shift in SAR interferometry," Geoscience and Remote Sensing, IEEE Transactions on, vol. 32, no. 4, pp. 855-865, July 1994.

[17] A. M. Guarnieri and S. Tebaldini, "ML-Based Fringe-Frequency Estimation for InSAR," IEEE Geoscience and Remote Sensing Letters, vol. 7, no. 1, pp. 136-140, Jan 2010.

[18] R. Bindschadler, H. Choi, A. Wichlacz, R. Bingham, J. Bohlander, K. Brunt, H. Corr, R. Drews, H. Fricker, M. Hall, R. Hindmarsh, J. Kohler, L. Padman, W. Rack, G. Rotschky, S. Urbini, P. Vornberger, and N. Young, "Getting around Antarctica: new high-resolution mappings of the grounded and freely-floating boundaries of the
Antarctic ice sheet created for the International Polar Year," The Cryosphere, vol. 5, no. 3, pp. 569-588, 2011. [Online]. Available: http://www.the-cryosphere.net/5/569/2011/

[19] G. Krieger, A. Moreira, H. Fiedler, I. Hajnsek, M. Werner, M. Younis, and M. Zink, "TanDEM-X: A Satellite Formation for High-Resolution SAR Interferometry," IEEE Transactions on Geoscience and Remote Sensing, vol. 45, no. 11, pp. 3317-3341, Nov 2007.

[20] I. R. Joughin, R. Kwok, and M. A. Fahnestock, "Interferometric estimation of three-dimensional ice-flow using ascending and descending passes," IEEE Transactions on Geoscience and Remote Sensing, vol. 36 , no. 1, pp. 25-37, Jan 1998.

[21] O. Lang, B. T. Rabus, and S. Dech, "Velocity map of the Thwaites Glacier catchment, West Antarctica," Journal of Glaciology, vol. 50, no. 168, pp. 46-56, 2004. 